Plant Tissue Cult. \& Biotech. 23(1): 59-66, 2013 (June)

$\overline{\mathrm{PTC} \& \mathrm{~B}}$

\title{
Evaluation of Genetic Relationship between Trigonella- Melilotus complex Using CCMP Markers
}

\author{
Amandeep Hora and C.P. Malik* \\ School of Life Sciences, Jaipur National University, Jaipur, Rajasthan, India \\ Key words: CCMP, DNA fingerprinting, Genetic correlation, Melilotus, Trigonella
}

\begin{abstract}
To distinguish the taxa at the specific and varietal levels a range of DNA fingerprinting techniques are being employed. The objective of the current study was to establish the genetic correlation between the Trigonella-Melilotus complex i.e. M. indica, M. alba and Trigonella polyceratia using ten Consensus Chloroplast Microsatellite Primers (CCMPs). The polymor-phism between the two genera indicated that they are intimately related and symbolize novel incongruity. Owing to less significant level of genetic variation, detected through CCMP, the differences between the two genera could be accredited to gene mutation or inconsequential chromosomal alterations.
\end{abstract}

\section{Introduction}

The legume family is the third largest family of angiosperms with approximately 730 genera and over 19400 species distributed world-wide. The Papilionoideae has received the most attention, because it is the largest and most widespread of the three legume subfamilies with an estimated 476 genera and 13860 species (Wojciechowski et al. 2004). For instance, the level of coumarin in some species of Trigonella is nearly same as that found in most species of Melilotus. Many species of Trigonella are attacked by the sweetclover (Melilotus) weevil, Sitona cylindricollis Fahr.

Linnaeus (1753) classified Melilotus as one group under Trifolium. Seringe (1825) placed the two genera Melilotus and Trigonella in a special section known as Grammocarpus of genus Trigonella. Bhattacharyya (1958) concluded that Trigonella and Melilotus might be treated as two subgenera. Various DNA fingerprinting techniques have been used to differentiate the taxa at the specific and varietals levels. These include RAPD and ISSR (Abdel 2009, Bussell et al. 2005). Both furnish very good taxonomic markers to determine the pattern of changes occurring in DNA. Further chloroplast specific micro satellites are used

*Author for correspondence. 
to assess the maternal and paternal plastid inheritance (Cato and Richardson 1996, Basha and Sujatha 2009, Karnawat et al. 2013), evaluation of interspecific polymorphism and the detection of hybridization and introgression and phylogeny of plant population.

Melilotus, known as Melilot or Sweet-clover, belongs to tribe Trifolieae and is a genus of the family Fabaceae. It comprises about 20 species originally from Europe and Asia, it is now distributed world-wide mainly in the temperate and subtropical regions of Europe, Asia and North Africa (Polhill and Raven 1981). According to Sharma and Tiagi (1979) two species occur in Jaipur (Rajasthan) and these are Melilotus alba and M. indica. The two species are distinguishable on the basis of length of inflorescence at fruiting stage and color of corolla.

Exclusive of the interference of environmental conditions, molecular markers can quickly and precisely genetic differences which are more dependable than morphological or biochemical methods. To explore chloroplast variation, universal primers targeted to mononucleotide repeats occurring in chloroplast genome is a useful tool. Moreover, chloroplast specific microsatellites have been used to assess maternal and paternal plastid inheritance (Cato and Richardson 1996) and appraisal of interspecific polymorphism.

In addition to assessing genetic relationship among the species, CCMPs are used to evaluate the organelle specific primer polymorphism (Hora and Malik 2012), identification of maternal and paternal parents and phylogeny of plant populations (Karnawat et al. 2013).

The objective of the present study was to evaluate the effectiveness of CCMP marker in determining the genetic relationships between the two genera Melilotus and Trigonella.

\section{Materials and Methods}

Fresh, young leaves were sampled from the plants of the two genera collected from the gram fields adjacent to Jaipur National University, Jaipur, and main campus in the month of February, 2012 and were recognized morphologically. These leaves were used for ensuing molecular biology studies.

DNA extraction: DNA isolation was performed on five grams of leaf tissue ground in liquid nitrogen. Total genomic DNA was extracted individually from younger leaves of species following the standard CTAB method with minor modifications by the addition of $2 \%$ sodium meta bisulphate (Doyle and Doyle 1990). DNA concentrations were determined electrophoretically versus known amount of $\lambda$ DNA as standards. For PCR, DNA samples were adjusted to a concentration of $5 \mathrm{ng} / \mu \mathrm{l}$. 
CCMP primer analysis: A set of ten CCMP primers specific to chloroplast genomes of dicotyledonous angiosperms (Weising and Gardner 1999) were used for the characterization of organellar genome of two genera i.e., Trigonella and Melilotus. The PCR amplifications were carried out in a $10 \mu \mathrm{l}$ reaction mixture containing $5 \mathrm{ng}$ of genomic DNA, 1x PCR buffer (10 mM Tris pH 9.0, $50 \mathrm{mM} \mathrm{KCl}$ and $\left.1.5 \mathrm{mM} \mathrm{MgCl}_{2}\right), 0.4 \mu \mathrm{M}$ each of forward and reverse primers, $150 \mu \mathrm{M}$ of each dNTPs and 0.6 Units of Taq DNA polymerase (Bangalore Genei, India). PCR amplifications were performed in GeneAmp 9700 thermal cycler (Perkin Elmer Applied Biosystems) with initial denaturation at $94^{\circ} \mathrm{C}$ for 4 min; 35 cycles of denaturation at $92^{\circ} \mathrm{C}$ for $30 \mathrm{~s}$, annealing at $55^{\circ} \mathrm{C}$ for $30 \mathrm{~s}$ and extension at $72^{\circ} \mathrm{C}$ for $45 \mathrm{~s}$; and a final extension at $72^{\circ} \mathrm{C}$ for $5 \mathrm{~min}$. The amplified PCR products were resolved by electrophoresis on $4 \%$ agarose (Bangalore Genei, India) gel and visualized by ethidium bromide staining. Banding pattern was recorded under ultraviolet light and documented in Alpha Innotech Fluorchem gel documentation system. As the amplification products that resulted from organelle specific markers were run on agarose gels.

Data analysis: The reproducible bands were scored and entered as binary traits ("1" for presence and "0" for absence). Genetic similarity was computed based on jaccard's similarity coefficient using NTSYS-Pc program (Rolf 2002). Further the similarity matrix was subjected to cluster analysis by the UPGMA. The percentage of polymorphism was calculated as the proportion of polymorphic bands with the total number of bands.

\section{Results and Discussion}

Chloroplast specific microsatellites are used to assess the maternal and paternal plastid inheritance (Cato and Richardson 1996), evaluation of interspecific polymorphism and the detection of hybridization and introgression and phylogeny of plant population. For details one may refer to Basha and Sujatha (2009). With a view to assessing genetic relationships among the two species, to evaluate the organelle specific primer polymorphism, and identification of maternal and consensus chloroplast microsatellite primers were used. Ten primers specific to chloroplast genome gave amplification products. All the CCMP primers except ccmp 8 and 9 yielded a single, discrete PCR product.

Allele sizes of amplification products (bp) from two different genera generated by consensus chloroplast microsatellite primers (CCMP1 to 10) are set in Table 1. Very diminutive difference was observed in allele size of the two different genera with CCMP1, 3 and 5. The two different genera appear to be genetically similar. The largest allele size of amplification product generated by CCMP was with CCMP10 (190 bp) and the smallest is with CCMP 2 (70 bp). All 
other primers gave amplification product, which was comparable with that of Argemone and tobacco species.
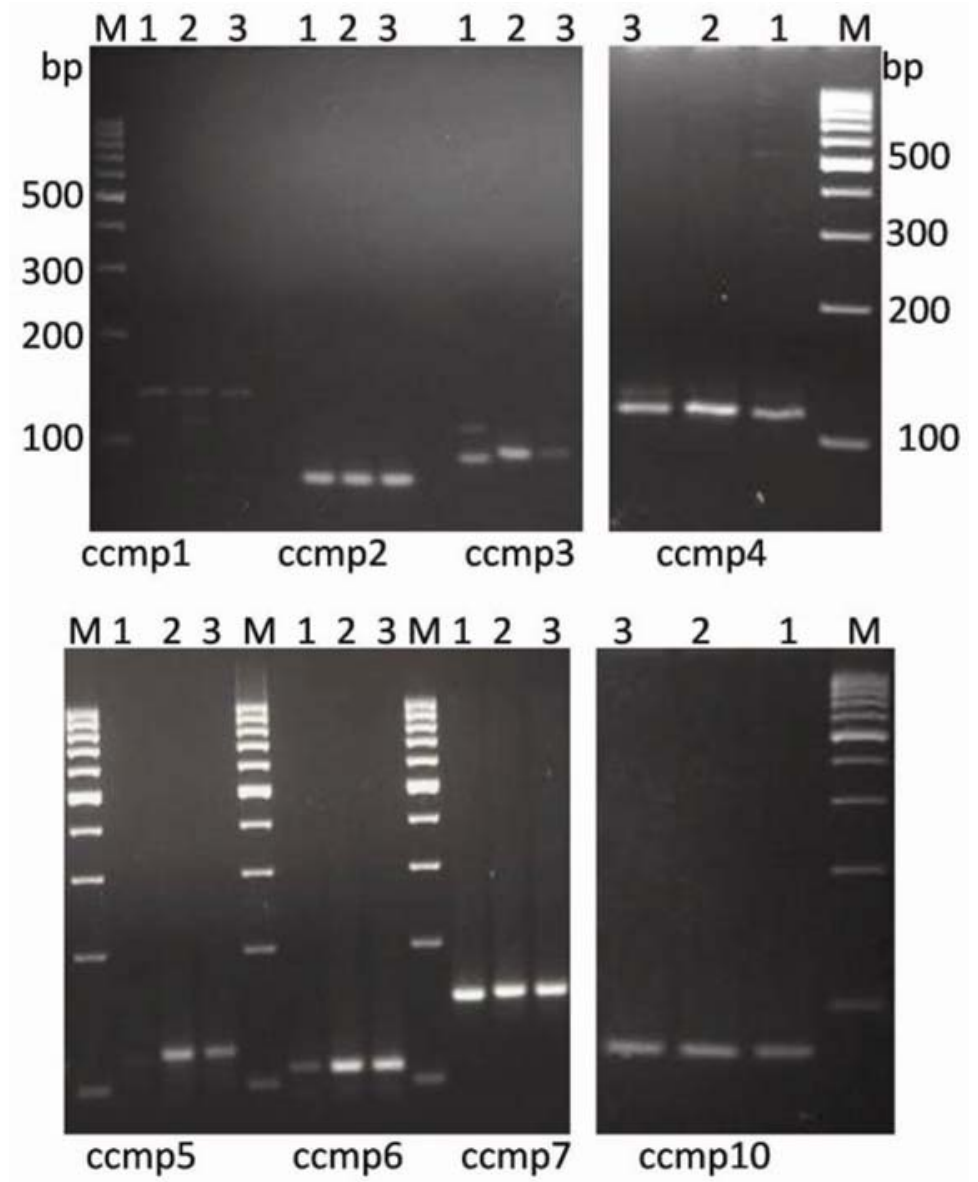

Fig. 1. PCR profiles of the M. indica, M. alba and Trigonella polyceratia generated by different CCMP primers. M - Marker 1. Melilotus incdica, 2. Melilotus alba and 3. Trigonella polyceratia.

Melilotus is so closely related to Trigonella that it is extremely difficult to separate the two genera. On the basis of cytological data and from the point of view of external morphology, Bhattacharyya concluded that Melilotus and Trigonella were very closely related that the two genera might be treated as two subgenera under one genus.

Cluster analysis indicated the segregation of T. polyceratia from the other two Melilotus species at 0.45 (Fig. 2, Table 2). Further at 0.847 M. parviflora and M. alba are nearly similar. Apparently, the two genera appear to be isolated. 
Heyn (1966) reported the presence of intermediate species between the three genera Medicago, Trigonella and Melilotus. Therefore, some species of Medicago viz. M. ruthenica and M. polycarpa are considered by most botanists to be intermediate between Trigonella and Medicago. In fact, Medicago supported a monophyletic group distinct from that formed by Melilotus, which is included within Trigonella and Trifolium basal to the remainder of Trifolieae. The present investigations bring out the significance of ccmp in discriminating among the disputed taxa, and are in accordance with the studies of Basha and Mulpuri (2009).

Table 1. Allele size of amplification products generated by consensus chloroplast microsatellite primers (CCMP1 to 10).

\begin{tabular}{lcccccccc}
\hline Species & \multicolumn{7}{c}{ Size of amplification product (bp) } \\
\cline { 2 - 9 } primer & Ccmp1 & Ccmp2 & Ccmp3 & Ccmp4 & Ccmp5 & Ccmp6 & Ccmp7 & Ccmp10 \\
\hline T. polyceratia & 140 & 70 & 80 & 130 & 135 & 120 & 160 & 190 \\
& & & 102 & & & & & \\
M. parviflora & 129 & 70 & 82 & 130 & 137 & 120 & 160 & 190 \\
& 140 & & & & & & & \\
M. alba & 140 & 70 & 82 & 130 & 137 & 120 & 160 & 190 \\
A. Mexicana & 139 & 240 & 112 & 150 & 100 & 95 & 150 & 115 \\
N. tabacum & 139 & 189 & 112 & 126 & 121 & 103 & 133 & 103 \\
\hline
\end{tabular}

CCMP
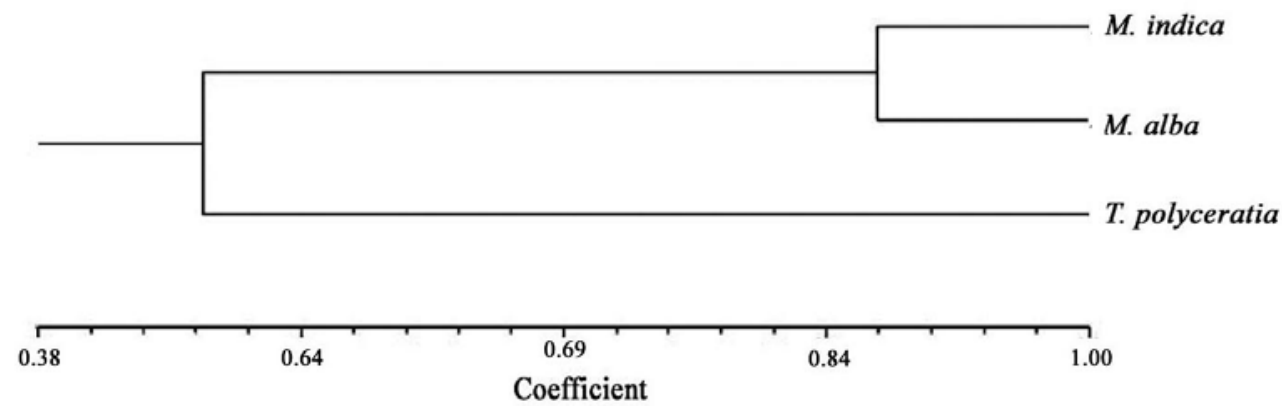

Fig. 2. Dendrogram based on genetic distance computed from UPGMA in the three species.

The present analysis indicates that T. polyceratia is linked with Melilotus. Marzauk and El-Bakataushi (2011) based on RAPD analysis suggested that $T$. polyceratia is more closely related to Medicago sativa than $T$. foenum graecum. Interestingly, Kawashty et al. (1998) inferred close affinity between T. foenum graecum and T.polyceratea on the basis of flavonoid profiles. According to these workers T. polyceratia needs to be retained in the genus Trigonella. Marzouk 
(2006) and Ahmad and Marzauk (2002) confirmed inclusion of T. polyceratia under Medicago on the basis of their protein profiles, morphology and anatomical data. Earlier Bena (2001) suggested inclusion of T. polyceratia under Medicago based on the sequence of two ribosomal transcribed spaces (ITS1, ITS2) and external transcribed spaces (ETS).

Table 2. Jaccard's similarity coefficient.

\begin{tabular}{lccc}
\hline & M. indica & M. alba & T. polyceratia \\
\hline M. indica & 1.00 & & \\
M. alba & 0.87 & 1.00 & \\
T. polyceratia & 0.45 & 0.50 & 1.00 \\
\hline
\end{tabular}

Chloroplast specific microsatellites are used to assess the maternal and paternal plastid inheritance (Cato and Richardson 1996), evaluation of interspecific polymorphism and the detection of hybridization and introgression and phylogeny of plant population. With a view to assessing genetic relationships among the two species, to evaluate the organelle specific primer polymorphism, and identification of maternal and consensus chloroplast microsatellite primers were used.

Present investigations have attempted to examine the level of genetic variation within Trigonella-Melilotus complex. The two marker systems have been employed to evaluate genetic diversity and to seek deep insight on the phylogenetic relationship of two genera. The data from the present study should be seen as a basis for future researches with a view to defining the level of genetic relationship. Present investigation has also given significant indicators in unravelling genotype relationship and this may further help in developing and planning breeding program.

Present molecular studies unequivocally aid in the establishment of genetic relationships between Trigonella, Melilots and Medicago and clarify their inscrutable genetic relationships. Present authors propose that the genus Trigonella belongs to tribe Trifolieae, subtribe Trigonellinae of the subfamily Papilionoideae. This subtribe should also include three other related genera e.g. Medicago, Melilotus and Trifolium along with Trigonella as proposed by Bena (2001) and Dangi et al. (2004).

\section{References}

Abdel AMIA (2009) Isozymes, RAPD and ISSR Variation in Melilotus indica (L.) All. and M. siculus (Turra) B.G. Jacks. (Leguminosae). Academic Journal of Plant Sciences 2(2): 113-118. 
Evaluation of Genetic Relationship between Trigonella- Melilotus

Ahmed MF and Marzouk RI (2002) A numerical study on the genus Trigonella L. (Leguminosae) in Egypt. Proc. 2nd Int. Conf. Biol. Sci. (ICBS) Fac. Sci. Tanta University 2: 189-222.

Basha SD and Sujatha M (2009) Genetic analysis of Jatropha species and interspecific hybrids of Jatropha curcas using nuclear and organelle specific markers. Euphytica 168: 197-214.

Basha SD, Francis G, Makkar HPS, Becker K and Sujatha M (2009) A comparative study of biochemical traits and molecular markers for assessment of genetic relationships between Jatropha curcas L. germplasm from different countries. Plant Science 176: 812823.

Battacharyya NK (1958) A comparative study of the cytology of few species of two allied genera, Trigonella and Melilotus. Caryologia 11: 165-180.

Bena G (2001) Molecular phylogeny supports the morphologically based taxonomic transfer of the Medicagoid Trigonella species to the genus Medicago L. Plant. Syst. Ecol. 229: 217-236.

Bussell JD, Waycot M and Chappill JA (2005) Arbitrarily amplified DNA markers as characters for phylogenetic inference perspect. Plant Ecol. Evol. Syst. 7:3-26.

Cato SA and Richardson TE (1996) Interspecific and intraspecific polymorphism at chloroplast SSR loci and the inheritance of plastids in Pinus radiate Don. Theoretical and Applied Genetics 9: 587-592.

Dangi RS, Lagu MD, Choudhary LB, Ranjekar PK and Gupta VS (2004) Assessment of genetic diversity in Trigonella foenum-graecum and Trigonella caerulea using ISSR and RAPD markers. BMC Plant Biology 4:13.

Doyle J and Doyle JL (1990) Isolation of plant DNA from fresh tissue. Focus. 12:13-15.

Heyn CC (1966) An evolutionary study of fruit morphology in the tribe Trigonelleae (Leguminosae). J. Phytomorphology 18:54-59.

Hora A and Malik CP (2012). Assessment of genetic relationship between Melilotus albus and M. indica (L.). All using PCR-RAPD and ccmp markers. Jour. Plant Sci. Res. 28: 105-111.

Karnawat M, Hora A and Malik CP (2013) The use of RPAD and ISSR markers for genetic relationship of two Argemone species and natural 'hybrids'. J. Plant Sci. Res. 29(1): 91-102.

Kawashty SA, Abdalla MF, Gamal El Din EM and Saleh NAM (1998) The chemosystematics of Egyptian Trigonella species. Biochem. Sys. Ecol. 26: 851-856.

Linnaeus C (1753). Facsimile ed. 1, Royal Society, London. Species Plantarum 2.

Marzouk RI (2006) Seed protein analyses as a support to the transfer of Trigonella cylindracea Desv. and T. polyceratia (L.) Trautv. to genus Medicago L. Taeckholmia. 26: 17-33.

Marzouk RI and El-Bakatoushi R (2011) Assessment of relocation of Trigonella cylindraceae L. and T. polyceratia (L.) Trautv. to genus Medicago as inferred by RAPD and RFLP analyses. Pak. J. Bot. 43(5): 2289-2294.

Polhill R and Raven P (1981) Royal Botanical Gardens Advances in Legume Systematics Part 1. 
Rolf FJ (2002) NTSYS-Pc: Reference Manual Exeter publishing Ltd., New York.

Seringe D (1825) Melilotus Tourn. In DC. Prodromus systematis naturalis, Paris 2: 171-185.

Sharma S and Tiagi B (1979) Flora of North Eastern Rajasthan Kalyani publishers New Delhi 235: 2789.

Weising K and Gardner RC (1999) A set of conserved PCR primers for the analysis of simple sequence repeat polymorphism in chloroplast genomes of dicotyledonous angiosperms. Genome 42: 9-19.

Wojciechowski MF, M Lavin and MI Sanderson (2004) A phylogeny of legumes (Leguminosae) based on analysis of the plastid matK gene resolves many wellsupported subclades within the family. Amer. J. Bot. 91: 1846-1862. 